\title{
A Critical Need for National Guidelines
}

\author{
Susanne Osanto ${ }^{a}$ Hein van Poppel \\ aDepartment of Clinical Oncology, Leiden University Medical Center, The Netherlands \\ ${ }^{b}$ Department of Urology, Catholic University of Leuven, Belgium
}

In this months' issue of ONKOLOGIE, Christoph Rölling and co-workers describe the rigorous and time-consuming developmental process which resulted in the interdisciplinary evidence based German S3 guidelines on diagnosis and treatment of prostate cancer [1]. The process encompassed a huge amount of work and was initiated by the German Society of Urology (DGU). The efforts took place over a period of several years, started in 2005 and were finalized and presented in September 2009. Rölling et al. provide detailed information concerning the composition of the group of different professional stakeholders, disciplines, involved in the development process, including members of the steering group, the research team involved in selecting, reviewing and rating the evidence, and individuals involved in formulating the final recommendations. Experts from 10 different medical societies or organizations, the Center for Quality in Medicine (Ärtzliches Zentrum fur Qualität in de Medizin, ÄZQ) and a patient organization were involved in the development of the guidelines. This process required several meetings over the years and additional re-evaluation steps. With this detailed report the authors adequately deal with the increasing concern about the quality, reliability and independence of practice guidelines since information on the methodological quality of the guidelines developed by specialty societies has not always been available [2].

In the past, guideline development has been largely based on expert opinion, and the views and opinions of other stakeholders [3]. In Germany, before 2005, apparently only one high quality, evidence based medicine (EBM) guideline existed (referred to as S3 according to the definition of the Scientific Medical Societies in Germany) and until then German guidelines in Urology were exclusively based on expert consensus of 'evidence' (level S1/S2). This asked for evidence based guidelines since various differences in the health care system limit the applicability of international evidence based guideline recommendations while the language barrier was perceived as an obstacle for implementation. The team made use of existing guidelines of AUA, EAU, NICE, and EBRO in The Netherlands, next to the Cochrane library and PubMed for systematic reviews.

Worldwide there is a need for guidance of clinicians in their daily work when faced with dilemmas and the need to take appropriate decisions. Physicians in university and community-based hospitals have become dependent on continuous medical education and clear and concise guidelines which help guide them to keep up with the ever growing number of clinical reports. The clinical landscape is constantly changing since patient populations at time of diagnosis change due to changes in lifestyle, new imaging techniques for diagnosing evolve, whereas novel treatment options including operational procedures and therapeutic compounds appear rather fast at the horizon. Practice guidelines clearly offer practical solutions to clinicians and need to be frequently updated to be useful to clinicians. According to Shekelle et al. [4], as a general rule, guidelines should be reassessed for validity every 3 years. A quick update process of a 'living guideline' is foreseen by the current steering committee.

Guidelines are more likely to be adopted as long as there is a strong professional support and if the implementation does not result in increased healthcare costs. The German guidelines involved a wide range of experts, although this apparently did not always make the task easier. The public consultation before final editing of the guidelines is a remarkable and potentially valuable approach to keep close contact with the basis. This approach may preclude potential unwillingness of the patients to undergo a certain treatment and thus result in enhanced adherence to the guidelines developed. It would be of interest to obtain more detailed information why (only) 5 out of 23 comments received from the public consultation period were adopted.

\section{KARGER}

Fax +497614520714

Information@Karger.de

www.karger.com (c) 2010 S. Karger GmbH, Freiburg

Accessible online at:

www.karger.com/onk
Susanne Osanto, MD, PhD

Department of Clinical Oncology

Leiden University Medical Center

Albinusdreef 2, 2333 ZA Leiden, The Netherlands

Tel. +31 71 526-3464, Fax -6760

s.osanto@lumc.nl 
Interestingly, of the 12,899 hits screened, only 420 publications were included as source of evidence underscoring that the vast majority of literature is not useful for evidence based medicine regimens. However, in our opinion, small studies and valid clinical observations may still be highly relevant under certain circumstances notwithstanding the compelling evidence in favor of adhering to level-one evidence recommendations. Evidence based medicine applies to 'groups of people' and one should not discount the 'importance of clinical expertise'.

Some questions remain after reading the article. Were initial recommendations substantially changed after the validation by the total group by telephone conferences and daily meetings and what criteria have been applied (e.g. costs, logistics, ethics). It is not clear whether costs of different methods of diagnosis and treatment have been taken into account in the ultimate recommendations. Were adaptations made in the initial recommendations based on the clinical directness and applicability in the German society, knowing that extrapolating data used in a clinical situation which differs from the original clinical study situation may lead to major differences in outcome for the patient. Thus, information about whether and to what extent the recommendations were modified by taking into account not only the quality of the existing data in the literature, but also such other variables would have given more insight in the quality of the ultimate recommendations. The future now lies in investment in the dynamics of guidelines required in an ever changing landscape and to test what percentage of recommendations are indeed implemented or diverted from in daily practice.

Thus, the next task for the DGU after the finalization of the guidelines on prostate cancer is the assessment of the impact on daily practice and to monitor adherence to their guidelines produced by subspecialty medical societies and general medical societies in order to measure the true value on health care. In principal, guidelines and evidence-based medicine aims for the ideal that health care professionals make conscientious use of current best evidence in their everyday practice, but adherence to guidelines is known to be rather variable as has been shown for the guidance issued by the National Institute for Clinical Excellence (NICE) in the UK [5]. Alongside with the continuous evolution of health care technologies, evidence based medicine and practice guidelines, the limitations of EBM guidelines have also become apparent and critics of EBM argue that guidelines are most appealing to health economists, policymakers and managers, for measuring performance and deciding on financial resources.

The question remains why each country should prepare their own national guidelines instead of sharing data which have emerged and been already published elsewhere. For the current German guidelines, guidelines and recommendations in other countries and from other organizations have been used. Clearly, practical recommendations may be influenced by local circumstances, specialist density, doctors' training, availability of imaging and radiotherapy equipment, national insurance and coverage policies and other differences between health care systems in different countries. However, the adoption of common standards should improve the consistency and quality in the reporting of guideline development worldwide and provide a framework to encourage international comparison of clinical practice guidelines.

Joining already established international collaboration of researchers and policy makers who seek to improve the quality and effectiveness of clinical practice guidelines by establishing a shared framework for their development, reporting and assessment, may be the next step.

\section{Conflict of Interest}

The authors do not have any conflict of interests.

\section{References}

1 Röllig C, Nothacker M, Wöckel A, Weinbrenner S, Wirth M, Kopp I, Ollenschläger G, Weißbach L: Development of the Interdisciplinary EvidenceBased S3 Guideline for the Diagnosis and Treatment of Prostate Cancer: Methodological Challenges and Solutions. Onkologie 2010;33:396-400.

2 Grilli R, Magrini N, Penna A, Mura G, Liberati A Practice guidelines developed by specialty societies: the need for a critical appraisal. Lancet 2000;355:103-106.
3 Browman GP: Development and aftercare of clinical guidelines: the balance between rigor and pragmatism. JAMA 2001;286:1509-1511.

4 Shekelle PG, Ortiz E, Rhodes S, Morton SC, Eccles MP, Grimshaw JM, Woolf SH: Validity of the Agency for Healthcare Research and Quality clinical practice guidelines: how quickly do guidelines become outdated? JAMA 2001;286:14611467.
5 Sheldon TA, Cullum N, Dawson D, Lankshear A, Lowson K, Watt I, et al.: What's the evidence that NICE guidance has been implemented? Results from a national evaluation using time series analysis, audit of patients' notes, and interviews. BMJ 2004;329:999-1006. 